\title{
Logic and Ontology
}

\author{
Nino B. Cocchiarella
}

\begin{abstract}
A brief review of the historical relation between logic and ontology and of the opposition between the views of logic as language and logic as calculus is given. We argue that predication is more fundamental than membership and that different theories of predication are based on different theories of universals, the three most important being nominalism, conceptualism, and realism. These theories can be formulated as formal ontologies, each with its own logic, and compared with one another in terms of their respective explanatory powers. After a brief survey of such a comparison, we argue that an extended form of conceptual realism provides the most coherent formal ontology and, as such, can be used to defend the view of logic as language.
\end{abstract}

Logic, as Father Bochenski observed, developed originally out of dialectics, rules for discussion and reasoning, and in particular rules for how to argue successfully. ${ }^{1}$ Aristotle, one of the founders of logic in western philosophy, described such rules in his Topics, where logic is presented in this way. Indeed, the idea of logic as the art of arguing was the primary view of this subject in ancient philosophy. It was defended in particular by the Stoics, who developed a formal logic of propositions, but conceived of it only as a set of rules for arguing. ${ }^{2}$

Aristotle was the founder not only of logic in western philosophy, but of ontology as well, which he described in his Metaphysics and the Categories as a study of the common properties of all entities, and of the categorial aspects into which they can be analyzed. The principal method of ontology has been one or another form of categorial analysis, depending on whether the analysis was directed upon the structure of reality, as in Aristotle's case, or upon the structure of thought and reason, as, e.g., in Kant's Critique of Pure Reason. Viewed in this way, the two

\footnotetext{
${ }^{1}$ See $[\mathrm{L} \& O]$, p. 278.

${ }^{2}$ See, e.g., [L\&O], p. 282.
} 
subjects of logic and ontology could hardly be more different, and many schools in the history of philosophy, such as the Stoics, saw no common ground between them. Logic was only a system of rules for how to argue successfully, and ontology, as a categorial analysis and general theory of what there is (in the physical universe), was a system of categories and laws about being.

Scholastic logicians also drew a sharp distinction between logic and ontology, taking the latter to be about "first intentions" (concepts abstracted directly from physical reality), and the former about "second intentions" (concepts abstracted wholly from the "material" content of first intentions, as well as about such categorial concepts as individual, proposition, universal, genus, species, property, etc., and so-called syncategorematic concepts such as negation). According to Aquinas, second intentions have a foundation in real entities, but "exist" only in knowledge; i.e., they do not exist in the real world but depend on the mind for their existence - which is not say that they are subjective mental entities. ${ }^{3}$

Aristotle left us not one but two very different logics, however; namely, the early dialectical logoi of the Topics, and the formal syllogistic logic of the Prior Analytics, a later work, which, according to Bochenski, treats logic essentially the way that contemporary mathematical, symbolic logic does; namely, as "dissociated from dialectic", i.e. not as "an art of thought" ([L\&O], p. 285). Indeed, according to Bochenski, the new mathematical, symbolic logic is "a theory of a general sort of object" (ibid.), so that "logic, as it is now conceived, has a subject matter similar to that of ontology" (ibid., p. 288).

The idea that logic has content, and ontological content in particular, is described today as the view of logic as language. This view is generally rejected in favor of a view of logic as an abstract calculus that has no content of its own, and which depends upon set theory as a background framework by which such a calculus might be syntactically described and semantically interpreted. ${ }^{4}$ We briefly describe the opposition between these two views of logic in section one, as well as give some of the history of the idea of logic as language. In section two, we argue that predication is more fundamental than membership and that different theories of predication are ontologically based on different theories of universals, the three most prominent types being nominalism, realism, and conceptualism. These theories of universals can be developed as alternative formal ontologies, each with its own logic, and, in that regard, each with its own account of the view of logic

\footnotetext{
${ }^{3}$ See $[\mathrm{L} \& \mathrm{O}]$, p. 283.

${ }^{4}$ See van Heijenoort [1967] for a description of these two views of logic, and for a criticism of the view of logic as language.
} 
as language. The opposition between the views of logic as language and logic as calculus can be mitigated in this way by using set theory as a mathematical framework in which different formal ontologies can be described and compared with one another in terms of their explanatory powers, even if only in terms of a somewhat distorting external semantical representation within set theory. We then briefly examine nominalism, logical realism, and conceptualism within the framework of comparative formal ontology and argue that an extended form of conceptual realism seems to provide the most coherent formal ontology by which to defend the view of logic as language.

\section{Logic as Language versus Logic as Calculus}

The relation between logic and ontology today, according to Bochenski, is that ontology is "a sort of prolegomenon to logic" (ibid., p. 290). That is, whereas ontology is an intuitive, informal inquiry into the categorial aspects of entities in general, "logic is the systematic, formal, axiomatic elaboration of this material predigested by ontology" (ibid.). In addition to this difference in method, i.e. of ontology being intuitive and informal, and logic being formal and systematic, there is also the difference that whereas "ontology as it is usually practiced is the most abstract theory of real entities, logic in its present state is the general ontology of both real and ideal entities," i.e. of abstract as well as concrete entities (ibid.).

Bochenski's example of the general ontology of the new logic is type theory, which, he says, is "strikingly similar to the old ontological views about 'being'," (ibid., p. 287), specifically that being is not a genus, because in type theory being is not univocal but is systematically ambiguous. ${ }^{5}$ Type theory is not the dominant paradigm of logic today, however; and, in fact, the idea of logic having any content at all, no less as containing a general ontology, is generally rejected in favor of the view of logic as calculus, which, as noted, is the dominant view today. Logic, on this view, is an abstract calculus devoid of any content of its own, but which can be given varying interpretations over varying domains of arbitrary cardinality, where the domains and interpretations are all part of set theory. Accordingly, if ontology really is a prolegomenon to logic, then, on this view, it can only be represented

\footnotetext{
${ }^{5}$ It is noteworthy that Bertrand Russell, the founder (in 1908) of type theory, originally held that being is univocal in his earlier (1903) Principles of Mathematics. For an account of Russell's development from a univocal to a systematically ambiguous notion of being, see Cocchiarella [1987], chapter one.
} 
as part of set theory. That is, it is only in the different set-theoretic domains and interpretations that ontology as "a theory of a general sort of object" can be said to be part of logic. It is not type theory, in other words, but set theory that contains a general ontology and that represents the dominant view of logic today. In fact, according to some proponents of this view, all philosophical analyses, and not just those that are part of ontology, are to be carried out within definitional extensions of set theory, i.e. in set theory with the possible addition of concrete objects (urelements) and empirical predicates. ${ }^{6}$

Bochenski rejected the view of logic as calculus, i.e., of logic as "a sort of game" the statements of which "do not and cannot pretend to be true in any meaning of the term" (ibid., p.276). A formal system or calculus in which logical constants are distinguished from nonlogical (descriptive) constants, and in which logical axioms and rules are distinguished from nonlogical axioms and rules, is not devoid of content, in other words, i.e., it is not a merely formal system, as the view of logic as calculus would have it. Rather, it is a logistic system in which logic is a language with content of its own. Moreover, as a general framework by which to represent our commonsense and scientific understanding of the world (through the introduction of descriptive constants and nonlogical axioms), the logical forms of a logistic system are syntactic structures that, as it were, carry their semantics on their sleeves. It is by assigning such logical forms to the (declarative) sentences of a natural language or a scientific theory that we are able to give logically perspicuous representations of the truth conditions of those sentences, and thereby locate them ontologically within our general conceptual framework. In this regard, a sufficiently rich formal logic is the basis of a lingua philosophica within which conceptual and ontological analyses can be carried out, and therefore a framework for general ontology. This approach, in contrast to the view of logic as calculus with set theory as the framework for general ontology, is what is meant by the view of logic as language.

The idea of a lingua philosophica goes back at least to Descartes and Leibniz, and perhaps even to the speculative grammarians of the 12th century who believed that there was one grammar underlying all of the natural languages of humanity. ${ }^{7}$ The speculative grammarians did not develop a formal logic as the basis of such a grammar, however. They believed that its structure was determined

\footnotetext{
${ }^{6}$ See "On the nature of cetain philosophical entities" in Montague [1974] for a description and defense of this view of set theory. Also, see Cocchiarella [1988], section 1, for a discussion and criticism of this view.

${ }^{7}$ Cp. Küng [1967].
} 
by real things in the world and that a philosopher could discover that structure only by considering the ontological nature of things. Descartes also believed that underlying all languages was a lingua philosophica; but what it represented was the form of reason and not the nature of things in the world. Such a language would contain a mathesis universalis, but its construction must await an analysis of all of the contents of consciousness into the simple ideas that were their ultimate constituents.

Leibniz also thought that a universal language exists underlying all natural languages, and that such a language represented the form of human reason. He called the framework for such a philosophical language a characteristica universalis, and took it as having three main goals. ${ }^{8}$ The first was that the universal character could serve as an international auxiliary language that people of different countries and cultures could use to communicate with one another. This goal is not part of the view of logic as language today. The second and third goals, on the other hand, are central to the idea of a logistic system. The second goal was that the universal character was to be based on an ars combinatoria, an idiography or system of symbolization, by means of which a logical analysis could be given of all of the actual and possible concepts that might arise in science. Such an ars combinatoria would contain both a theory of logical form-i.e. a theory of all of the possible forms that meaningful expressions might have - and a theory of definitional forms, i.e. a theory of the operations whereby new concepts can be constructed on the basis of given concepts. The third goal of the universal character was that it must contain a calculus ratiocinator, a complete system of deduction that would characterize valid argument forms, and that could be used to study the logical consequences of what was already known. Also, once a universal character was constructed, Leibniz thought that it could be used as the medium for a unified encyclopedia of science, in which case it would then also amount to a characteristica realis, a representational system that would enable us to see into the inner nature of things. In this way, the universal character would not only contain a general ontology, but also the more specific ontologies of each field of science as well.

Though Leibniz did construct some fragments of a calculus ratiocinator, nothing like an adequate system fulfilling his ideal was constructed until Frege's (1879) Begriffsschrift, which Frege extended in his (1893) Grundgesetze der Arithmetik by adding to it his theory of value-ranges (Werverläufe), or extensions of concepts and relations, as abstract objects. This latter theory was in effect a theory about

\footnotetext{
${ }^{8}$ See Cohen [1954] for a more detailed account of Leibniz's project.
} 
how classes (Begriffumfangen) as the extensions of the concepts that predicates stand for in their role as predicates can be "grasped" by starting out from the concepts themselves, namely, by nominalizing the predicates and treating them as abstract singular terms that have the extensions of the concepts as their denotata. ${ }^{9}$

Here in Frege's extended version of his concept-script we have a paradigm of logic as containing a general ontology of both real and ideal objects. Indeed, Frege himself was quite explicit in maintaining that his concept-script was "not a mere calculus ratiocinator, but a lingua characteristica in the Leibnizian sense". ${ }^{10}$ His goal was to construct not just an abstract calculus but "a logically perfect language" that could be used as a general framework for science and mathematics. It was not to serve the purposes of ordinary natural language, as Leibniz's goal of an international auxiliary language was, but was intended as a tool for the analysis of concepts and the formal development of mathematical and scientific theories. The relation between his concept-script and ordinary natural language, according to Frege, was like that between a microscope and the eye. The eye is superior to the microscope in "the range of its possible uses and the versatility with which it can adapt to the most diverse circumstances", but "as soon as scientific goals demand great sharpness of resolution, the eye proves to be insufficient". ${ }^{11}$ In other words, just as the microscope is a device "perfectly suited" to the demand for great sharpness of visual resolution in science, so too the concept-script is "a device invented for certain scientific purposes, and one must not condemn it because it is not suited to others" (ibid.).

Unfortunately, Frege's logic, as extended to include a theory of value-ranges as abstract objects, was subject to Russell's paradox, which involves the mechanism of nominalization that Frege introduced to represent value-ranges as the extensions of concepts. ${ }^{12}$ The addition of the theory of value-ranges was an important and novel step, to be sure, because, as noted above, it was in this way that Frege was able to explain our "grasp" of abstract objects in terms of the concepts that predicates stand for; and that we can have such a "grasp" was essential to Frege's logicism, i.e. his reduction of number theory to logic. Still, it is important to note, the theory of value-ranges was not part of Frege's original concept-script, which

\footnotetext{
${ }^{9}$ See Cocchiarella [1987], Chapter Two, for a detailed defense of this interpretation of Frege's logic.

${ }^{10}$ Frege [1972], p. 90.

${ }^{11}$ Frege [1879], p. 6.

${ }^{12}$ See Cocchiarella [1987], Chapter Two, for a detailed analysis of what is involved in the derivation of Russell's paradox in Frege's logic.
} 
amounted in effect to the first formulation of (a version of) standard second-order logic. ${ }^{13}$ Frege himself was quite explicit in noting that "we can treat the principal part of logic without speaking of classes, as I do in my Begriffsschrift". ${ }^{14}$

It is not necessary that a nominalized predicate denote the extension of the concept that the predicate stands for in its role as a predicate in order to derive Russell's paradox. The paradox is derivable, in other words, even if, nominalized predicates, as abstract singular terms, are taken to denote the intensions of the concepts that predicates stand for - which, for Russell, were none other than the concepts themselves. Russell's way out of his paradox was the theory of types, where predicates are divided into a hierarchy of different types, and nominalized predicates of a given type can occur as argument expressions only of predicates of higher types. ${ }^{15}$ It was this division of predicates and their nominalizations that resulted in the systematic ambiguity of being in type theory, and other than as a way of avoiding paradox, it does not seem to be based on any deep insight into the nature of reality. For this reason, the theory of logical types is sometimes said to be an ad hoc system of logic.

There are other problems with type theory as well. Concrete objects, for example, are assigned only to the initial type of "individuals", which means that in order to construct the natural numbers (as higher-order objects) Russell had to assume that there are infinitely many concrete (non-abstract) "individuals". ${ }^{16}$ This was an unwarranted ontological assumption about the physical world that led to some dissatisfaction with the theory of types, especially among those who viewed it as an ad hoc way to avoid the paradoxes. Also, for Russell, the "individuals" of lowest type are events, and physical objects of both the micro- and macro-physical world are "logical constructions" from events, which means that physical objects are abstract and not concrete entities, contrary to our normal on-

\footnotetext{
${ }^{13}$ Here, by standard second-order logic, we mean the second-order logic that is complete with respect to Henkin general models - not the second-order logic that is incomplete with respect to so-called "standard" set-theoretic models. To claim that Frege's logic is incomplete is to confuse the (iterative) hierarchy of sets with Frege's hierarchy of concepts where Cantor's theorem fails (for reasons connected with Frege's double-correlation thesis, which we describe in section 4).

14 "Letter to Jourdain" (1910), in Bochenski [1961], p.360.

${ }^{15}$ Russell's division of predicates actually involves two hierarchies, one a "vertical" hierarchy of levels, and the other a "horizontal" hierarchy in which all of the concepts on a given level are ramified. See Cocchiarella [1987], Chapter One, for a description of these hierarchies and a detailed account of Russell's development of the theory of logical types.

${ }^{16}$ In Russell's earlier 1903 framework in which being is a genus, all entities are "individuals". It was only in his later theory of types that he used the word 'individual' to refer to objects of lowest type.
} 
tological intuitions about the world. Other ontological assumptions, such as the reducibility axiom and the axiom of choice, were also needed, and led to further dissatisfaction. ${ }^{17}$ In time, the theory of types was given up by most philosophers, as well as by mathematicians, in favor of set theory, which seems far more simple and intuitive in its assumptions about the existence of sets. The idea that there is an infinite set consisting, for example, of the empty set, $\varnothing$, singleton the empty set, $\{\varnothing\}$, doubleton the empty set and its singleton, $\{\varnothing,\{\varnothing\}\}$, and so on ad infinitum, does not depend on there being any concrete objects at all, and the assumption of its existence seems intuitively natural. There is no need for a reducibility axiom in set theory, moreover, and, given that sets are abstract objects that exist independently of all constructions of the mind, there seems to be no problem with assuming an axiom of choice for sets.

Finally, the development of formal, model-theoretic semantics by Tarski and others as a part of set theory led to many important results that fit very naturally with the logic as calculus view. ${ }^{18}$ As a result of this type of semantics, logic, as we have said, is generally viewed today as an abstract calculus with no content of its own, a calculus that can be assigned different interpretations over varying settheoretic domains, thereby resulting in what seems to many to be a very natural, formal explication of the important notions of logical consequence and logical truth. Indeed, these semantical developments in set theory, which in itself is a very powerful and useful framework for the development of mathematics, is taken by many contemporary philosophers and logicians to be the coup de grace for the view of logic as language.

\section{Predication Versus Membership}

Notwithstanding the great power and utility of set theory as a mathematical theory, and of set-theoretic model theory in particular as a method for proving a number of results in formal semantics, it is not the right sort of framework in which to represent either a general ontology or our commonsense and scientific

\footnotetext{
${ }^{17}$ The ramification of the different levels of type theory seemed to be nullified, according to Quine and other philosophers, by the reducibility axiom. Ramification also suggested that type theory represents a constructive theory of abstract entities, and the axiom of choice seems counter to the idea of such a constructive view.

${ }^{18}$ See Addison, Henkin, and Tarski [1965] for a number of results in this area. Also, see Hintikka [1988] for a discussion of how these results connect with the view of logic as calculus, as well as a defense of this view.
} 
understanding of the world. Membership, the basic notion upon which set theory is constructed, is at best a pale shadow of predication, which, in one form or another, is the basic notion upon which thought, natural language, and the logical forms of the view of logic as language are constructed. Indeed, so basic is predication that different theories of logical form as different versions of the view of logic as language are really based on alternative theories of predication. Traditionally, these alternative theories have been informally described as theories of universals, the three major types of which are nominalism, conceptualism, and realism. Here, by a universal we mean that type of entity that can be predicated of things, which is essentially the characterization originally given by Aristotle. ${ }^{19}$ As described by Porphyry in his Introduction to Aristotle's Categories, the three major types of theories of universals are concerned either with predication in language (nominalism), predication in thought (conceptualism), or predication in reality (realism). It is, in each case, the predicable nature of a universal that constitutes its universality, its one-in-many nature, and, at least in conceptualism and realism, that predicable nature is taken to be a mode of being that, unlike sets (of the iterative hierarchy) is not generated by its instances, and, in that sense, does not have its being in its instances, the way that sets have their being in their members. That is why sets should not be confused with universals, as has become all too common by those who take set theory as the only proper framework for philosophy.

That there are different theories of universals means, we have said, that there are different theories of predication, and, on the view of logic as language, this means that there can be alternative theories of logical forms - i.e. alternative formal logics - that can be taken as formal representations of different theories of universals, and, in that regard, as formal ontologies. Here, in the recognition that there can be alternative formal logics in the sense of a formal ontology-i.e. alternatives that can be compared and contrasted in various respects with one another - we find a clear rejection of the idea that the views of logic as calculus and logic as language are mutually exclusive. There is no inconsistency in the idea that the informal, intuitive theories of universals that have been described and proposed throughout the history of philosophy are each in its own way "the predigested material of ontology", and that the different versions of this material can be systematically developed and explained in terms of the methodology of modern symbolic logic by formulating each as a formal theory of predication that can be taken as the basis of both a formal logic and a formal ontology. Set theory and model-theoretic semantics, subject to the proper constraints dictated

\footnotetext{
${ }^{19}$ See Aristotle's De Interpretatione 17 a 39 for this acount of universals.
} 
by each theory of universals, can be used as a mathematical framework by which to construct and compare these different formal ontologies - but, and this cautionary note is important, only in the sense of providing an external, mathematical model of the ontology that each purports to represent in its own internal way.

Just as the construction of a particular theory of universals as a formal ontology will lend clarity and precision to our informal ontological intuitions, so too a framework of comparative formal ontology can be developed so as to provide clear and precise criteria by which to judge the adequacy of a particular formal ontology, and by which we might be guided in our comparison and evaluation of different proposals for such systems. This is not to deny the "validity" of each formal ontology as a correct perspective on reality, and in particular this does not mean that the "truth" of any formal ontology is merely a "relative truth" of no objective ontological significance. Rather, it is only by constructing and comparing different formal ontologies in the general framework of comparative formal ontology that we can make a rational decision about which system we should ourselves ultimately adopt.

\section{The Vagaries of Nominalism}

The connection between ontology and logic as a theory of logical form is stronger and also somewhat different from that between an informal scientific theory (such as, e.g., classical, or relativistic, particle mechanics) and an axiomatic version of that scientific theory as an applied form of a logistic system, i.e. as an applied theory of logical form. Ontological distinctions are not formally represented by descriptive predicates and the axioms regarding how they relate to one another, but by the logico-grammatical categories of a theory of logical form and the rules and axioms governing their possible transformations. In frameworks other than nominalism, these categories are based ultimately on an intuitive, informal distinction between modes of being. In a system in which being is not univocal but multivalent, for example, there will be variables of different logical types corresponding to the logico-grammatical categories that are taken to represent the different modes of being of that theory, and when bound by quantifiers that are interpreted ontologically rather than substitutionally these variables are assumed to have the corresponding entities of that type as their values. Given our assumption that a formal ontology is based on a theory of universals, which is represented by a formal theory of predication, the two principal types of variables in question here are predicate and individual variables. We will in general restrict ourselves 
to considering just these types of variables and the views regarding their analyses as ontological categories.

In nominalism, the basic ontological thesis is that there are no universals beyond the predicate expressions, or the tokens of such, that occur in language. This means that either there are no predicate variables and quantifiers binding such, or if there are, then they must be interpreted only substitutionally, and hence that certain constraints must be imposed on predicate quantifiers. ${ }^{20}$ The only variables that are allowed in nominalism to be bound by quantifiers having an ontological interpretation, in other words, are individual variables, which means that being, in nominalism, is not multivalent but univocal. For nominalism, predication is just predication in language, which, semantically, is explained as a relation between predicate expressions and the objects they are true of (or satisfied by). It is in this sense that nominalism, or what Bochenski calls logical nominalism, maintains that "logic is about language" ([L\&O], p. 292).

Ontological nominalism, according to Bochenski, claims that "there are no ideal entities" (ibid.), which reduces to the claim that there are no universals (beyond predicate expressions) if that is all that is meant by an "ideal entity". To be sure, the medieval form of nominalism seemed to preclude all abstract entities; but that might be because universals were the only abstract entities that were then at issue. In any case, let us call that type of nominalism in which ideal, or abstract, entities of any kind are rejected traditional ontological nominalism.

In modern ontological nominalism, at least of the sort described by Nelson Goodman, nominalism "does not involve excluding abstract entities, ... but requires only that whatever be admitted as an entity at all be counted as an individual", where for a system "to treat entities as individuals... is to take them as variables of the lowest type in the system". ${ }^{21}$ This, essentially, is what we described above as logical nominalism, where only individual variables are allowed to be bound by quantifiers having an ontological interpretation. It is not traditional ontological nominalism, however, because it allows ideal, or abstract, entities to be values of the bound individual variables. Goodman himself, in The Structure of Appearance, took qualia, which are ideal entities of a phenomenalist ontology, to be the basic individuals of his own nominalist formal ontology. On the other hand, Goodman does reject sets (or classes in the mathematical sense of the iterative hierarchy) as admissible values of the individual variables of a nominalist system;

\footnotetext{
${ }^{20}$ As shown in Cocchiarella [1989a], the constraints for nominalism turn out to be just those of standard predicative second-order logic, which can be extended to ramified second-order logic.

${ }^{21}$ Goodman [1956], p.17.
} 
but that is because the generating relation of set theory, namely membership, allows us to distinguish sets that are made up of the same urelements (or "atoms" of the constructional system in Goodman's terminology). For example, where $a$ is an urelement (or atom), i.e. an object such that nothing is a member of it, $\{a\}$ and $\{a,\{a\}\}$ are different sets even though both are $(\in-)$ generated from $a$. For Goodman, the nominalist's dictum that rules this kind of ontology out is: "No distinction of entities without distinction of content", that is, "no two distinct things can have the same atoms". ${ }^{22}$ Thus, on Goodman's explication, modern ontological nominalism does preclude some kinds of ideal entities (e.g. sets, in particular) other than universals, though it also allows others, such as qualia.

Quine, together with Goodman, once attempted to construct a nominalist system that satisfied Goodman's nominalist dictum. ${ }^{23}$ But it was a temporary gesture, and he returned to his preferred ontological framework of set theory-but only as formulated within first-order logic, where to be is to be the value of a bound individual variable, which Quine later preferred to call an "objectual" variable instead. Other than violating Goodman's dictum of "no distinction of entities without a distinction of content," Quine's preferred framework of set theory comes close to being a form of modern ontological nominalism, though Quine himself calls his ontology platonistic and refers to sets as universals.

Quine's understanding of his ontology as platonistic and of sets as universals is based on a rather involuted argument, the essentials of which are as follows: if we were to adopt platonism as a theory of universals as represented by a higher-order logic in which predicate as well as individual variables can be bound, then (1) predicate quantifiers can be given a referential ontological interpretation only if predicates are (mis)construed as singular terms (i.e. terms that can occupy the argument or subject positions of predicates); and (2) assuming extensionality, (3) predicates, as singular terms, can only denote sets, which (4) must then also be the universals that are the values of the predicate variables in predicate positions; and therefore (5) predication must be the same as membership, in which case (6) we might as well replace predicate variables by individual variables (thereby accepting nominalism's exclusion of bound predicate variables) and take sets as values of the individual variables, arriving thereby at (7) a first-order theory of membership (set theory), which (8) is platonist because it has abstract entities as

\footnotetext{
${ }^{22}$ Goodman [1956], p. 21.

${ }^{23}$ See Goodman and Quine [1947]. This paper opens with a clear renunciation of abstract entities altogether.
} 
values of its one type of variable. ${ }^{24}$ Thus, beginning with higher-order logic with bound predicate variables as a version of platonism, we arrive at the nominalist position to recognize only quantification with respect to individual variables (or the subject positions of predicates), but with individual variables that can have abstract sets as their values, which are therefore really universals (i.e. entities that have a predicable nature).

Without going into the details here, it is noteworthy that Frege would reject (1), accept (2), accept (3) as applied to value-ranges, and reject (4)-(7). Russell would accept (1), reject (2) and (3), accept (4), and reject (5)-(7). Goodman, as we have noted, would reject (8) in so far as it applies to such ideal entities as qualia. Quine's implicit argument, needless to say, can hardly be taken as a paradigm of how one should view the relation between logic and ontology. Nevertheless, it does indicate how one can adopt an ontological view of logic, and yet end up with a system that coincides in all other respects with the view of logic as calculus.

\section{The Vindication (Almost) of Logical Realism}

The paradigm of a formal logic in which all logico-grammatical categories represent ontological categories is the system of Frege's Grundgesetze. The ontological insight that is fundamental to this logic is Frege's distinction between saturated and unsaturated entities, where all and only saturated entities are "complete objects" in a sense analogous to Aristotle's notion of primary substance - though Frege's "complete objects" include abstract objects, such as propositions (Gedanken) and value-ranges, as well as concrete, physical objects, whereas only physical objects count as primary substances in Aristotle's ontology. Unsaturated entities are functions of different ontological types, depending on the types of their arguments and the types of their values. For example, first-level concepts (Begriffe), which Frege also called properties (Eigenshaften), are functions from objects to truth values, and second-level concepts, such as those represented, e.g., by the universal and existential quantifiers, are functions from first-level concepts to truth values.

Predication in Frege's formal ontology is explained in terms of the unsaturated nature of functions; that is, the nexus of predication for Frege is just a type of functionality. This is a mathematical interpretation, not essentially different from the set-theoretic one in terms of membership; for, whereas membership in a set can

\footnotetext{
${ }^{24}$ See Quine [1963], p. 257, for what amounts to the essentials of these claims. Also, see Cocchiarella [1992] for a more detailed account of these claims and their sources in Quine's writings, as well as of this argument that is implicit in those writings.
} 
be explained in terms of functionality (i.e. in terms of the characteristic function of a set that assigns 1 to its members and 0 to its nonmembers), functionality can in turn be explained in terms of membership. Of course, unlike functions, sets do not have an unsaturated nature; but then the only explanation Frege ever gave of the unsaturated nature of a function turned on the unity of a sentence (which is based on the unsaturated nature of a predicate expression as a linguistic function) and the unity of the proposition (Gedanke) expressed by a sentence. Regarding the unsaturated nature of the predicate of a sentence, for example, Frege claimed that "this unsaturatedness ... is necessary, since otherwise the parts [of the sentence] do not hold together" ([PW], p.177). Similarly, regarding the unsaturated nature of the nexus of predication in a proposition, Frege claimed that "not all parts of a thought [Gedanke] can be complete; at least one must be 'unsaturated', or predicative; otherwise they would not hold together" (Frege [1952], p. 54). Thus, although predication is explained in Frege's ontology in terms of functionality, functionality seems ultimately to presuppose the notion of predication. If predication had really been taken as basic in Frege's ontology, and functionality explained in terms of predication, then perhaps functionality would be essentially different from membership after all.

This, in fact, is the situation in Russell's ontology, where functionality is explained in terms of predication and the unity of a proposition. A function, according to Russell, is just a many-one relation, where a relation, as the nexus of predication of a proposition - i.e. as the relating relation of that proposition, as opposed to a relation occurring as a "term" of the proposition - is what explains the unity of the proposition. ${ }^{25}$ What holds the constituents of a proposition together, according to Russell, is a relation relating those constituents in a certain way, i.e. a relation as the nexus of predication of that proposition, which, because the proposition "exists" independently of language and thought, amounts to a form of predication in reality - but of course a reality that includes such abstract entities as propositions. ${ }^{26}$ Unlike Frege, however, Russell (at least until 1913) took properties and relations to be objects, i.e. entities that could themselves be related by relations (of a higher order/type) in the nexus of predication; and, as a result, he had to reject the idea that properties and relations are unsaturated,

\footnotetext{
${ }^{25}$ Cp. $[\mathrm{PoM}]$, p. 83 . See also p. 43 where the word 'term' is said to be synonymous with 'individual' and 'entity'.

${ }^{26}$ Russellian propositions (at least after Russell dropped his 1903 theory of denoting concepts) are sometimes also taken to be states of affairs, and states of affairs are assumed to be a fundamental part of reality in many ontologies.
} 
i.e. that the predicative nature of properties and relations consist in their having an unsaturated nature. ${ }^{27}$ But then this leaves Russell with no ontological explanation of the difference between a relation occurring as the relating relation of a proposition as opposed to its occurring merely as a "term" of that proposition - a situation that could in principle lead to something like Bradley's infinite regress argument against this kind of account of the unity of a proposition.

There is also some irony in the fact that, although Russell rejected the idea of unsaturated concepts, the "vertical" part of his ramified theory of types was initially suggested to him (and in that regard motivated) by a hierarchy of "levels" of unsaturated concepts and relations that Frege was committed to in his ontology, but which Frege did not in fact incorporate in his formal logic. ${ }^{28}$ Frege's commitment was clear because, as a consequence of its unsaturated nature, a function had to be of a different (and in a sense of a "higher") ontological level, than that of its arguments. The ground level of this ontological hierarchy consists, of course, of all and only "complete" (saturated) objects. The first level "above" the ground level then consists of all of the first-level concepts and relations that have objects as their arguments; and the next level consists of all of the second-level concepts and relations, including not only the functions from first-level concepts and relations to truth values, but also unequal level relations between objects and first-level concepts and relations. Third-level, fourth-level, etc., concepts and relations similarly have objects and the concepts and relations of the preceding levels as their arguments. The result is a hierarchy that continues on through one ontological level after the other ad infinitum. ${ }^{29}$

Now contrary to the way that Russell understood predication at the different levels of his (vertical) hierarchy, the relation between first- and second-level concepts and relations (and, in general, between the $n$th and $(n+1)$ th levels of concepts and relations), which Frege described as a falling within relation, is not the same as that between objects and first-level concepts and relations, which Frege

\footnotetext{
${ }^{27}$ After 1914, under the influence of Ludwig Wittgenstein, Russell gave up the view of properties and relations as "objects", i.e. entities that could be "logical subjects" of relations. Russell did not seem to realize this meant that he had to give up the vertical hierarchy of his theory of types and restrict himself to ramified second-order logic. See Cocchiarella [1987], Chapter Five, for a detailed account of this change.

${ }^{28}$ See Cocchiarella [1987], Chapter Two, for an account of the relation beween Russell's theory of types and Frege's hierarchy of levels.

${ }^{29}$ There are other types of functions in Frege's ontology as well, it should be noted - such as functions from objects to objects other than truth values, or the function from first-level concepts and relations to their extensions as value-ranges.
} 
described as a falling under relation. That is why, unlike Russell's higher "level" properties and relations, Frege's second- and higher-level concepts and relations are not represented by predicates but by variable-binding operators, which, unlike predicates, can be commuted and iterated, as well as occur within the scope of one another. This hierarchy, in fact, is not based on anything like Cantor's power-set theorem; and, in fact, contrary to the hierarchy of sets determined by the latter, there are no more second-level concepts and relations in Frege's hierarchy than there are first-level concepts and relations (and, in general, no more $(n+1)$ th-level concepts and relations than there are $n$ th-level concepts and relations). Of course, given Frege's correlation of first-level concepts with their value-ranges, there are also no more first-level concepts and relations than there are objects.

What all this suggested to Frege was that third- and higher-level concepts and relations could all be represented in a way by second-level concepts and relations, and that therefore there was no need to explicitly deal with third- and higher-level concepts and relations in his formal logic. That is why Frege saw no point in introducing quantifiers (representing third-level concepts) for second-level concepts and relations. Indeed, he is quite explicit in assuming (what I have elsewhere called) a double-correlation thesis to the effect that all second-level concepts and relations can be correlated with and represented by first-level concepts and relations, which in turn can be correlated with and represented by their value-ranges. ${ }^{30}$ For example, in the monadic case, the thesis can be symbolized as follows:

$$
(\forall Q)(\exists F)(\forall G)[(Q x) G(x) \leftrightarrow F(G)],
$$

where ' $Q$ ' is a variable for second-level concepts, ' $F$ ' and ' $G$ ' are one-place predicate variables for first-level concepts, and the nominalization of a predicate (in this case ' $G$ ') is indicated by simply deleting the parentheses (and commas in the case of a relational predicate) that otherwise occur as part of the predicate in its role as a predicate. ${ }^{31}$

It is by means of this double-correlation that Frege explains "the miracle of number", i.e. the existence of numbers as objects, denoted by numerals and other

\footnotetext{
${ }^{30}$ See Frege's Grundgesetze der Aritmetik, section 25, for an explicit statement of this correlation. Also, see Cocchiarella [1987], Chapter Two, for a more detailed account.

${ }^{31}$ Frege uses the smooth-breathing operator to represent the nominalization of the predicate ' $G$ ', as in ' $\grave{\varepsilon} G(\varepsilon)$ ', which in ordinary language he reads as 'the concept $G$ ', but which denotes the extension of $G$. Frege took the longer phrase 'the extension of the concept $G$ ' as unnecessary in an extensional framework. (See Cocchiarella [1987], Chapter Two, for a fuller explanation of these issues.)
} 
singular terms. As saturated abstract objects, in other words, the natural numbers are "derived" from certain second-level unsaturated concepts, specifically, those that are represented by numerical quantifier phrases. For example, corresponding to the second-level concept represented by the quantifier phrase, 'there are 4 objects $x$ such that', which we can symbolize as ' $\exists^{4}$ ', there is a first-level concept $F$ such that a first-level concept $G$ falls within the second-level concept represented by ' $\exists^{4}$ ' if, and only if, the (extension of the) concept $G$ falls under $F$; in symbols:

$$
(\exists F)(\forall G)\left[\left(\exists^{4} x\right) G(x) \leftrightarrow F(G)\right] .
$$

Note that the extension of a concept $G$ falls under the first-level concept $F$ that is posited here if, and only if, there are four objects that have $G$, i.e. if, and only if, the extension has four members; and hence $F$ is really the concept under which all and only four-membered classes fall. The extension of the concept $F$ itself then is just the class of all four-membered classes, which on Frege's (and Russell's) analysis is just the number four as denoted by the numeral ' $4{ }^{\prime}{ }^{32}$ It is in this way, in other words, by going through a double-correlation and representation of second-level numerical concepts with first-level concepts, and similarly of first-level concepts with their extensions, that we are able to "grasp" the natural numbers as objects that can be denoted by numerals and other singular terms.

Now it is noteworthy that Frege's double-correlation thesis indicates a way by which Russell's paradox can be avoided. Indeed, there are two related ways involved here, and not just one. The first is simply to exclude from Frege's original ontology all unequal-level relations (such as the second-level relation of predication between an object and a first-level concept), which means that the resulting hierarchy of concepts and relations must now be homogeneously stratified. Frege's double-correlation thesis, extended to apply to all higher-level concepts and relations, can then be restricted to a correlation that is homogeneously stratified. In particular, using $\lambda$-abstracts for the generation of complex predicates, including those in which nominalized predicates occur as abstract singular terms, we can arrive at a consistent (relative to weak Zermelo set theory) reconstruction of Frege's logic by restricting the grammar to those $\lambda$-abstracts that can be homogeneously stratified. ${ }^{33}$ The homogeneously stratified comprehension principle for first-level

\footnotetext{
${ }^{32}$ Of course, on Russell's analysis the number 4 is a higher-order "object", and in fact there are infinitely many numbers 4 in Russell's type theory, one for each level of the vertical hierarchy greater than two.

${ }^{33} \mathrm{~A}$ formula or $\lambda$-abstract $\varphi$ is homogeneously stratified (or just $h$-stratified) iff there is an assignment $t$ of natural numbers to the terms and predicate expressions occurring in $\varphi$ (including
} 
concepts and relations then has the following form,

$\left(\mathrm{HSCP}_{\lambda}^{*}\right)$

$$
\left(\exists F^{n}\right)\left(\left[\lambda x_{1} \ldots x_{n} \varphi\right]=F\right)
$$

where the $\lambda$-abstract $\left[\lambda x_{1} \ldots x_{n} \varphi\right]$ is homogeneously stratified. From this (and Leibniz's law) follows the weaker, but more usual, comprehension principle,

$$
\left(\exists F^{n}\right)\left(\forall x_{1}\right) \ldots\left(\forall x_{n}\right)\left[F\left(x_{1}, \ldots, x_{n}\right) \leftrightarrow \varphi\right]
$$

where $\varphi$ is homogeneously stratified (which includes all the wffs of standard second-order logic, i.e. wffs in which no nominalized predicates occur as abstract singular terms). Russell's paradox, as represented by

$$
(\exists F)(\forall x)(F(x) \leftrightarrow(\exists G)[x=G \wedge \neg G(x)])
$$

cannot be derived from this principle (despite its being well-formed and therefore meaningful), because the comprehending formula in this case is not homogeneously stratified.

The resulting system, which is obtained by extending standard second-order logic by including nominalized predicates as abstract singular terms, is called $\lambda$ HST $^{*}$. This system can be shown to be equiconsistent with the theory of simple types. Unlike the latter, however, we can add an axiom of infinity here that is independent of how many, if any, concrete objects there are in the physical world.

The formal logic $\lambda \mathrm{HST}^{*}$ can be used as a logical reconstruction of the logic implicit in Russell's early framework as well as of Frege's logic, except that for the latter we would also add a principle of extensionality:

$$
\left(\forall x_{1}\right) \ldots\left(\forall x_{n}\right)(\varphi \leftrightarrow \psi) \rightarrow\left[\lambda x_{1} \ldots x_{n} \varphi\right]=\left[\lambda x_{1} \ldots x_{n} \psi\right]
$$

Of course, one could also add modal operators for necessity and possibility and in that way extend Frege's ontology to a modal variant as well, in which case $\left(\right.$ Ext $\left._{n}^{*}\right)$ would not be assumed as an (onto)logical truth or law.

$\varphi$ itself if it is a $\lambda$-abstract) such that (1) for all terms $a, b$, if $(a=b)$ occurs in $\varphi$, then $t(a)=t(b)$; (2) for all $n \geq 1$, all $n$-place predicate expressions $\pi$, and all terms $a_{1}, \ldots, a_{n}$, if $\pi\left(a_{1}, \ldots, a_{n}\right)$ is a formula occurring in $\varphi$, then (i) $t\left(a_{i}\right)=t\left(a_{j}\right)$, for $1 \leq i, j \leq n$, and (ii) $t(\pi)=t\left(a_{1}\right)+1$; (3) for $n \geq 1$, all individual variables $x_{1}, \ldots, x_{n}$, and formulas $\chi$, if $\left[\lambda x_{1} \ldots x_{n} \chi\right]$ occurs in $\varphi$, then (i) $t\left(x_{i}\right)=t\left(x_{j}\right)$, for $1 \leq i, j \leq n$, and (ii) $t\left(\left[\lambda x_{1} \ldots x_{n} \chi\right]\right)=t\left(x_{1}\right)+1$; and (4) for all formulas $\chi$, if $[\lambda \chi]$ occurs in $\varphi$ and $a_{1}, \ldots, a_{k}$ are all of the terms or predicates occurring in $\chi$, then $t([\lambda \chi]) \geq \max \left[t\left(a_{1}\right), \ldots, t\left(a_{k}\right)\right]$. 
The second way of avoiding Russell's paradox and reconstructing Frege's logic is not to exclude unequal-level relations, but to allow - as Frege himself noted in an appendix to his Grundgesetze - that "there are cases where an unexceptional concept has no class answering to it as its extension" ([G\&B], p.235), i.e. that not every predicate expression when nominalized will denote (a value of the individual variables). All $\lambda$-abstracts, including those that are not homogeneously stratified, can be admitted as meaningful predicates that stand for a concept or relation, in other words, but not all will also necessarily denote an object when nominalized. This means that the first-order part of the logic must be "free of existential presuppositions", so that although we have a comprehension principle that applies to all $\lambda$-abstracts,

$$
\left(\exists F^{n}\right)\left(\left[\lambda x_{1} \ldots x_{n} \varphi\right]=F\right),
$$

where $\left[\lambda x_{1} \ldots x_{n} \varphi\right]$ need not be homogeneously stratified, it does not follow that we also have

$$
(\exists y)\left(\left[\lambda x_{1} \ldots x_{n} \varphi\right]=y\right)
$$

where $y$ is an individual variable (not occurring free in $\varphi$ ). In particular, by Russell's argument, the Russell predicate when nominalized does not denote (a value of the individual variables),

$$
\neg(\exists y)([\lambda x(\exists G)(x=G \wedge \neg G(x))]=y)
$$

even though the same predicate, by $\left(\mathrm{CP}_{\lambda}^{*}\right)$, stands for a concept:

$$
(\exists F)([\lambda x(\exists G)(x=G \wedge \neg G(x))]=F) .
$$

All of the concepts and relations that are represented in the first reconstruction, $\lambda \mathrm{HST}^{*}$, can be consistently assumed to have objects (e.g., extensions given $\left(\mathrm{Ext}_{n}^{*}\right)$, or intensions if (Ext $\left.{ }_{n}^{*}\right)$ is rejected) in this alternative reconstruction of Frege's logic, which (for that reason) we call $\mathrm{HST}_{\lambda}^{*}$, and which can also be shown to be equiconsistent with $\lambda \mathrm{HST}^{*}$ and therefore with the theory of simple types as well.

Note, however, that although the resulting logic can be taken as a reconstruction of Frege's logic and ontology, it cannot also be taken as a reconstruction of Russell's early ontology, because having rejected the notion of unsaturatedness and taken nominalized predicates to denote as singular terms the same concepts and relations they stand for as predicates, Russell cannot then allow that some predicates stand for concepts but, when nominalized, denote nothing. Still, there 
is the logical system $\lambda \mathrm{HST}^{*}$, which can be taken as a reconstruction of Russell's early ontological framework.

The upshot, accordingly, is that logical realism is not really defunct as either a logical or an ontological theory, and as a semantical framework for natural language it is in many respects actually superior to set theory. ${ }^{34}$ The idea of logic as language in the sense of logical realism is still very much alive, in other words, or at least can be resurrected and taken as an alternative to set theory as a semantical and ontological framework. Of course, there remains the problem in both Russell's and Frege's ontology of giving a philosophically coherent and satisfying account of predication. But then, no such account is forthcoming in set theory - unless one adopts Quine's mixture of nominalism and what he calls platonism. An account is forthcoming in conceptual realism, however, which includes an intensional as well as a natural realism as part of the ontology; and, in the intensional part, we can achieve most of what was attempted in logical realism without either the latter's platonism or its problem of giving a philosophically coherent account of predication. ${ }^{35}$ We will briefly review some of the main features of this third type of formal ontology in the remainder of this paper.

\section{Conceptualism Without a Transcendental Subject}

The principal method of ontology, we have noted, is categorial analysis. The major issue of such an analysis is how the different categories or modes of being fit together. In some ontologies, this issue is resolved by taking one of the categories or modes of being as "primary", with the others then explained as somehow dependent on that mode - as is the case, e.g., in Aristotle's moderate realism with its category of primary substances, and in Frege's logical realism with its category of "complete" (saturated) objects.

The different categorial analyses that have been proposed throughout the history of philosophy, we have also noted, have turned in one way or another on a theory of universals; and, such a theory, we have said, can be developed as a the-

\footnotetext{
${ }^{34}$ In Chierchia [1984] and [1985], $\lambda$ HST $^{*}$ is used as a semantical framework in Montague grammar that, at least for the semantics of gerunds, infinitives and other forms of nominalized predicates in natural language, is actually preferable to Montague's own type-theoretical intensional logic.

${ }^{35}$ See Cocchiarella [1996] for an account of conceptual realism as a formal ontology, and Cocchiarella [1992] for a comparison of this framework with Quine's views on set theory and the logic of classes.
} 
ory of the logical forms that perspicuously represent how the different categories fit together in the nexus of predication. We also noted that these theories have differed on whether the analysis of the fundamental forms of predication is to be based on the structure of reality or on the structure of thought and reason. Aristotle's analysis, for example, as well as that of the speculative grammarians of the 12 th century, was directed upon the structure of reality, whereas Descartes's and Leibniz's analyses were directed upon the structure of thought and reason. Kant's analysis of the categories of his Critique of Pure Reason was similarly directed on the structure of thought and reason, and is a paradigm of this sort of approach.

Unlike Aristotle's categories, which were based on physical objects as primary substances, Kant's categories were based on the notion of a judgment, and the different logical forms that judgments might have. There is no primary mode of being identified in this analysis, other than that of the thinking subject, whose "synthetic unity of apperception" is what unifies the categories in the different possible judgments that can be made. What categories there are and how they fit together is determined, according to Kant, by a "transcendental deduction", and the categories so deduced then form the basis of a transcendental logic. A similar approach was taken by Husserl who, in his phenomenological analyses, took logic and ontology to be based on a transcendental subjectivity. In both cases, the result is a conceptual idealism, the categorial structure of which is based on an assumed absolute a priori knowledge of the principles of a transcendental logic.

That a conceptual system is "transcendental" means that it is independent of our status as biologically, culturally, and historically determined beings, and hence independent of the laws of nature and our evolutionary history. Not all forms of conceptualism are committed to a transcendental view, however; nor must conceptualism in general be committed to idealism or a methodology that purports to be based on absolute a priori knowledge. Modern forms of conceptualism, for example, are usually based on a naturalized epistemology that, in one way or another, is not supposed to depend upon either Kant's synthetic unity of apperception or Husserl's transcendental subjectivity - which does not mean that other aspects of either Kant's or Husserl's analyses cannot be adopted on such a naturalized approach. Konrad Lorenz, for example, has described what might be called a biological Kantianism where the methodology is that of the empirical science of ethology, and where, instead of a transcendental deduction, the categories of thought are determined by evolution, which means that the results obtained cannot then be a form of absolute a priori knowledge. ${ }^{36}$ Similarly, Jean Piaget has

\footnotetext{
${ }^{36}$ See, e.g., Lorenz [1962].
} 
developed a genetic epistemology in which there are different stages of conceptual development both in the individual and in the history of knowledge, stages that are based on a "functional", as opposed to an absolute, a priori. ${ }^{37}$ There is no synthetic unity of apperception or transcendental subjectivity that is assumed in either of these approaches, it must be emphasized. But then there also seems to be no explicit account that explains the unity of thought and judgment, i.e., of predication in thought. A conceptual account of this unity must be given if conceptualism without a transcendental subjectivity is to be a viable alternative to nominalism and logical realism, especially as a basis for the view of logic as language.

An account is forthcoming, we maintain, once we allow for something like Frege's notion of unsaturatedness, but modified appropriately to explain the role of concepts in judgment and other forms of thought. Here, in conceptualism, we do not mean by a concept anything at all like what Russell or Frege meant, and in particular we do not mean a property or relation that can exist independently of the mind; but then neither do we mean a strictly subjective entity as well. Rather, by a concept in conceptualism we mean a certain type of intersubjectively realizable - and in that sense objective - cognitive structure, and in particular a cognitive capacity that can be exercised or realized at the same time by different people, as well as by the same person at different times. Predicable concepts, for example, are intersubjectively realizable cognitive capacities, or cognitive structures based on such capacities, for characterizing and relating objects in various ways. In the social context of learning a language, these capacities underlie our rule-following abilities in the correct use of predicate expressions, which means that they are the principal factors that determine the truth conditions of those expressions. It is in this way that the exercise of a predicable concept in a speech or mental act is what informs that act with a predicable nature.

The important point here is that as intersubjectively realizable capacities concepts are not images or ideas in the sense of particular mental occurrences (events), and in fact they are not objects of any sort at all. Rather, as cognitive capacities, concepts have an unsaturated nature that can be exercised by different people at the same time as well as by the same person at different times - and in fact some concepts might never be exercised at all (as, e.g., in the case of certain numerical concepts) without diminishing their status as capacities that could be exercised in appropriate contexts. Concepts are not occurrent states or events, in other words, but are rather like dispositions, except that, unlike dispositions, which

\footnotetext{
${ }^{37}$ Cf. Piaget [1972].
} 
have a "would-have" nature of being realized under suitable conditions, concepts have a "could-have" nature of being exercised in appropriate contexts. Of course, when exercised, concepts result in objects, namely, particular mental acts, such as judgments, and, when expressed overtly, certain types of speech acts, such as assertions or statements. Predicable concepts, as we have said, are what inform those acts with a predicable nature.

Conceptual thought consists not just of predicable concepts, however, but of referential and other types of concepts as well. Referential concepts, for example, are cognitive capacities that underlie our ability to refer (or purport to refer) to objects, and, as such, they too have an unsaturated cognitive structure. More importantly, referential concepts have a structure that is complementary to that of predicable concepts, so that each, when exercised or applied jointly in a basic mental or speech act, mutually saturates the other, resulting thereby in an act (event) that is informed with a referential and a predicable nature. It is the complementarity of predicable and referential concepts as unsaturated cognitive structures that is the basis of the unity of our thoughts and speech acts, and which explains why a transcendental subjectivity need not be assumed as the basis of this unity.

Every affirmative assertion (speech act) that is syntactically analyzable in terms of a noun phrase and a verb phrase (regardless of the complexity of either) is semantically analyzable, according to the kind of conceptualism we are describing here, in terms of an overt application of a referential and a predicable concept, and the assertion itself is the result of their mutual saturation in that joint application. It is this sort of joint application and mutual saturation of complementary cognitive structures that, in conceptualism, explains the nexus of predication in both thought and language. A speech act in which 'All swans are white' is asserted, for example, is the result of jointly applying the referential concept that 'all swans' stands for, which formally can be represented by ' $\forall x S w a n)$ ', with the predicable concept that 'is white' stands for, which formally can be represented by 'White( )', or, using $\lambda$-abstracts, by '[ $\lambda x W h i t e(x)]($ )'. The assertion can then be analyzed as having the logical form $(\forall x S w a n) W h i t e(x)$, or, equivalently, $(\forall x$ Swan $)[\lambda x W h i t e(x)](x)$, both of which perspicuously represent the cognitive structure of the assertion as the mutual saturation of the referential and predicable concepts that underlie it. Similarly, the cognitive structure of an assertion of 'Some swans are not white', where the negation is internal to the predicate, can be perspicuously represented as ' $(\exists x \operatorname{Swan})[\lambda x \neg$ White $(x)](x)$.

Singular reference, as in the use of a proper name or definite description, is not 
essentially different from general reference in conceptualism, which in many ways resembles the suppositio theory of medieval logicians. ${ }^{38}$ The syntactic category of names can in fact be taken to consist of common names and proper names as two proper subcategories, where proper names and most common names are taken to stand for sortal concepts. ${ }^{39}$ Sortal concepts are those cognitive capacities whose use in thought and communication is associated with certain identity criteria, i.e. criteria by which we are able to identify objects of the sort in question. The common name 'swan', for example, stands for a sortal concept by which we are able to identify and refer to swans, and a proper name, such as 'Aristotle', stands for a sortal concept by which we are able to identify and refer to a certain individual. In general, the use of a proper name brings with it the identity criteria of the most specific sortal common name associated with that proper name.

Singular reference is not essentially different from general reference, on this account, which means that the referential use of a proper name should also be represented by a quantifier phrase. This is particularly appropriate in that a proper name can be used without, as well as with, existential presupposition, and these different types of uses can be associated with the quantifiers $\forall$ and $\exists$, respectively. Thus, for example, '( $\exists x$ Aristotle)' can be used to represent a use of the proper name 'Aristotle' that is with existential presupposition - as, e.g., in an assertion of 'Aristotle is Greek', symbolized as ' $(\exists x$ Aristotle $)$ Greek $(x)$ '—whereas ' $(\forall x$ Pegasus $)$ ' can represent a use of the name 'Pegasus' that is without existential presupposition - as, e.g. in an assertion of 'Pegasus is winged', symbolized as ' $(\forall x$ Pegasus $)$ Winged $(x)$ '. The important point is that in both kinds of cases the referential concept is an unsaturated cognitive structure and not an "idea", image, or mental occurrent of any type, though, when exercised, the result is a mental occurrent (event) of some type or other. Definite descriptions can also be used without, as well as with, existential presuppositions; but, having explained this difference elsewhere, we will forego discussing them here. ${ }^{40}$

Referential concepts, as these examples indicate, are represented by quanti-

\footnotetext{
${ }^{38}$ Peter Geach, in $[\mathrm{R} \& \mathrm{G}]$, has criticized the suppositio theory as well as other theories of general reference. For a defense against Geach's arguments of the kind of conceptualist theory being proposed here, see Cocchiarella [1998].

${ }^{39}$ Common, as well as proper, names are different from predicates in that, as Geach has noted, they can be used "outside the context of a sentence" in "simple acts of naming" ([R\&G], p.52), i.e. acts that are not assertions and that do not in that regard involve the use of a name to refer. "Nouns in the vocative case used in greetings, and ejaculations like 'Wolf!' and 'Fire!' illustrate this independent use of names" (ibid.).

${ }^{40}$ See, e.g., Cocchiarella [1989b] and [1996].
} 
fier phrases, which formally can be symbolized as $(Q x S)$, where $Q$ is a quantifier symbol (such as $\forall$ or $\exists$, or a quantifier for 'most', 'few', etc.) indexed by the (individual) variable $x$, and $S$ is symbol for a common name, complex or simple. Complex common names, at least in English, are generated from more basic common names by attaching a (defining) relative clause to the latter. Formally, by adopting a new primitive operator '/, we can represent the operation of attaching a relative clause, represented by a formula $\varphi$, to a common name $S$, by the expression ' $S / \varphi$ ', which is read as ' $S$ (who, which) that is (are) $\varphi$ '. An assertion of 'Every citizen (who is) over twenty-one is eligible to vote', for example, can be symbolized as:

$$
(\forall x \text { Citizen } / x \text { is-over-21) }[\lambda x \text { Eligible-to-vote }(x)](x) .
$$

The truth conditions for sentences with complex names can be connected to the more usual kinds of truth conditions by such rules or meaning postulates as:

$$
\begin{gathered}
(\forall x S / \varphi) F(x) \leftrightarrow(\forall x S)[\varphi \rightarrow F(x)], \\
(\exists x S / \varphi) F(x) \leftrightarrow(\exists x S)[\varphi \wedge F(x)],
\end{gathered}
$$

and iterations of the /-operator can be reduced to simple conjunctive relative clauses by such laws as:

$$
\begin{aligned}
& (\forall x S / \varphi / \psi) F(x) \leftrightarrow(\forall x S / \varphi \wedge \psi) F(x), \\
& (\exists x S / \varphi / \psi) F(x) \leftrightarrow(\exists x S / \varphi \wedge \psi) F(x) .
\end{aligned}
$$

The important point here is that though these various formulas are logically equivalent, and therefore have the same truth conditions, they do not represent the same cognitive structures of our speech and mental acts. In conceptualism, our concern is not only with logical forms as perspicuous representations of the truth conditions of our assertions, but also with the logical forms that represent the cognitive structure of those assertions as well. It is this latter kind of representation that is essential to conceptualism's account of predication in thought and language.

\section{Conceptual Natural Realism and the Analogy of Being Between Natural and Conceptual Universals}

Without some associated form of realism, conceptualism is an ontology restricted to the conceptual realm, where it easily slips into the ontology of conceptual idealism, regardless whether or not the latter is based on a transcendental subjectivity. 
As a socio-biological theory of the human capacity for language and thought, however, conceptualism must presuppose some form of natural realism as the causal ground of that capacity. But then, natural realism must in turn presuppose some form of conceptualism by which to explain our capacity for language and thought, and in particular our capacity to form theories of the world and posit properties and relations as part of the causal order. Conceptualism and natural realism, in other words, presuppose each other as part of a more general ontology, namely, one or another form of conceptual natural realism. ${ }^{41}$ The form of conceptual naturalism we briefly describe here is itself part of a more general conceptual realism that includes an intensional realism of abstract objects as well, which we will describe section 7 .

Aristotle and Peter Abelard were conceptual natural realists (but not intensional realists), though they were not as clear as they could have been on the distinction between concepts and natural properties and relations. Abelard, in his Glosses on Porphyry, for example, does not distinguish the predicable concepts we exercise in thought from the natural properties that exist as common likenesses in things. A property (universal), according to Abelard, seems to "exist" in a double way, first as a common likeness in things (prior to, and independent of, our having any concept of that likeness), and then, through our capacity to abstract the likeness in things from our perception of them, as a predicable concept that "exists" somehow in our intellect. Aristotle also seems to describe natural kinds and properties in this double way, i.e. as having a mode of being both in things and then, through an inductive abstraction (epagoge), in the mind as well. Of course, it is possible that the problem of this "double existence" is really not the problem it has commonly been described to be; perhaps, for example, it is only a problem of explaining how the same predicate can stand for, or signify, both a concept and a natural property, where the latter only corresponds to, or is represented by, the former, i.e. where the two are not really the same universal after all. The point in any case is that concepts cannot literally be the same as the natural properties and relations they purport to represent, and in fact some concepts (especially for artifacts and social conventions) are not assumed to represent any natural properties or relations at all.

The distinction between concepts in the order of conception and natural properties and relations in the order of being does not mean that there must also be a

\footnotetext{
${ }^{41}$ Some of the differences between these forms depends on whether a constructive or holistic conceptualism is assumed, and whether the natural realism is part of an Aristotelian essentialism or not. See Cocchiarella [1989a] for a more detailed account.
} 
distinction between predicates that stand for concepts and predicates that stand for natural properties and relations. Rather, as indicated above, it is a matter of distinguishing between a primary and a secondary sense of signification. The same predicate, in other words, can be taken to stand for, or signify, a concept in the primary sense, and, in a secondary, derivative sense, also to stand for, or signify, a natural property or relation that corresponds to, or is represented by, that concept - so that it is not the same universal that can exist in a double way but a predicate that can signify in a double way instead. Similarly, in our theory of logical form, the same ( $n$-ary) predicate variables can be taken in a double way to have both ( $n$-place) concepts and ( $n$-ary) natural properties and relations as their values, so that the difference is not represented by different types of predicate variables but by the different types of predicate quantifiers that can be affixed to predicate variables. That is, the difference is a matter of the kind of second-order reference that is made by means of predicate quantifiers.

We can add to our conceptualist theory of logical form special quantifiers, $\forall^{n}$ and $\exists^{n}$, accordingly, that can be affixed to predicate variables, and that, when affixed, can be used to refer to natural properties and relations. Thus, for example, a fundamental thesis of natural realism is that every ( $j$-ary) natural universal is causally realizable, which formally can be stated as follows:

$$
\left(\forall^{n} F^{j}\right) \diamond^{c}\left(\exists x_{1}\right) \ldots\left(\exists x_{j}\right) F\left(x_{1}, \ldots, x_{j}\right),
$$

where the modal operator $\diamond^{c}$ represents a causal (or natural) possibility and not a logical or merely conceivable possibility. With the modal operator deleted, the thesis (NR) represents a form of Aristotle's moderate realism, which assumes that properties and relations "exist" only in re, i.e. only in the concrete, physical objects that have those properties and relations. With the modal operator (which indicates what is possible in nature), (NR) represents a modal moderate realism according to which natural properties and relations have a mode of being in the causal structure of the world, a mode that does not depend on whether or not there are objects having those properties and relations, and hence which is ante rem, but not (as in logical realism) independent of whether or not there could be such objects. ${ }^{42}$

\footnotetext{
${ }^{42}$ Many of the natural properties and relations of atoms and compounds now in existence were causally realizable when the universe was first formed but when there were no such atoms and compounds. Similarly, some properties of possible transuranic substances might never be realized because those kinds of atoms might never in fact exist, which is not to say that they could not be realized and do not figure in the causal structure of the world.
} 
Unlike concepts, which can be applied even in imaginary and fictional contexts that violate the laws of nature, natural properties and relations are "identical" when they are co-extensive as a matter of causal or natural necessity, a thesis that can be formulated as follows:

$$
\left(F^{j}={ }_{c} G^{j}\right)={ }_{d f} \square^{c}\left(\forall x_{1}\right) \ldots\left(\forall x_{j}\right)\left[F\left(x_{1}, \ldots, x_{j}\right) \leftrightarrow G\left(x_{1}, \ldots, x_{j}\right)\right] .
$$

It is "identity" in this sense that can be used to express the condition for when a natural property or relation "exists" corresponding to a given concept, i.e. for when the concept can be taken to represent such a property or relation. Thus, where a concept of a given domain is represented by a $\lambda$-abstract, $\left[\lambda x_{1} \ldots x_{j} \varphi\right]$, we can stipulate that there "exists" a natural property or relation corresponding to, and in that sense represented by, that concept as follows,

$$
\left(\exists^{n} F^{j}\right)\left(\left[\lambda x_{1} \ldots x_{j} \varphi\right]={ }_{c} F\right) .
$$

This principle is similar to the comprehension principle, $\left(\mathrm{CP}_{\lambda}^{*}\right)$, for concepts (as cognitive capacities), except that, instead of the strict identity, ' $=$ ', in $\left(\mathrm{CP}_{\lambda}^{*}\right)$, we use the restricted identity, ${ }^{\prime}={ }_{c}$ ', of having the same extension as a matter of a natural or causal necessity. However, unlike the comprehension principle for concepts, every instance of which is a logical or conceptual truth, an instance of the above principle can be stipulated only as a scientific hypothesis, and therefore only as an assumption that is subject to empirical confirmation or falsification.

Natural properties and relations are not intensional objects, it must be emphasized, nor are they objects of any other kind as well. As universals that can be realized in different places at the same time and that might have no instances at all in the world, natural properties and relations are not in the world the way that concrete objects are, nor can they be considered to have an "objectual" nature in any other sense as well. Rather, as causally determinate structures that are part the causal structure of the world, natural properties and relations have an unsaturated mode of being, which, although not the same as that of predicable concepts, can nevertheless be said to be analogous. We can conceptually grasp and understand the unsaturated mode of being of natural properties and relations, in other words, only as somehow analogous to the mode of being of concepts. Thus, just as predicable concepts do not exist independently of the human capacity for language and concept-formation, so too natural properties and relation do not exist independently of the causal structure of the world; and just as the laws of compositionality for concept-formation can be said to characterize the 
logical structure of the intellect as the basis for the human capacity for language and thought, so too the laws of nature regarding the causal connections between natural properties and relations, especially as structural aspects of natural kinds of things and stuff, can be said to characterize the causal structure of the world.

The reference to natural kinds here should not be confused with a reference to natural properties. For, in the kind of conceptual natural realism we have in mind here, natural kinds, when they are assumed to "exist", correspond not to predicable concepts, but to sortal concepts, i.e. the concepts represented by sortal common names. Here, by a natural kind we understand a type of causal structure, or mechanism in nature, that is the basis of the powers or capacities to behave in certain determinate ways that objects belonging to that natural kind have. According to Aristotelian essentialism, natural kinds are in fact the causal structures, or mechanisms in nature, that determine the natural laws of the different kinds of things there are, or can be, in nature. As such, a natural kind is not a "conjunction" of the natural properties and relations that objects belonging to that kind necessarily have, but rather is the causal ground or nexus of the events and states of affairs corresponding to such a conjunction. This ontological difference is analogous to the conceptual difference between sortal common-name concepts and predicable concepts and the way that referential concepts based on the former may be saturated by the latter in particular events or states of affairs. Thus, just as a referential concept based upon a sortal concept can be saturated by a predicable concept in a speech or mental act, so too a natural kind, is an unsaturated causal structure that, when realized by an object belonging to that kind, is saturated by the natural properties and relations of that object, resulting thereby in a nexus of events or states of affairs having that object as a constituent.

Accordingly, just as a predicate expression can signify both a predicable concept and a natural property or relation, a sortal common name can also signify or stand in a double way for both a concept and a natural kind as a causal structure, and sortal-name variables can be given a similar double interpretation as well. Thus, just as the quantifiers $\forall^{n}$ and $\exists^{n}$ can be affixed to predicate variables and enable us to refer to natural properties and relations, so too we can introduce special quantifiers $\forall^{k}$ and $\exists^{k}$, which, when affixed to sortal-name variables, enable us to refer to natural kinds. The logic of natural kinds can then be developed in terms of certain fundamental laws, such as the law that an object belongs to a natural kind only if being of that kind is essential to it, i.e. only if it must belong to that kind whenever it exists (as a real, concrete object). Where ' $E$ !' is a predicate constant for concrete existence (in an ontology where abstract objects 
can also "be", but not exist in this sense), this principle can formulated as follows:

$$
\left(\forall^{k} S\right)(\forall x S) \square^{c}[E !(x) \rightarrow(\exists y S)(x=y)] .
$$

Other principles that are part of the logic of natural kinds are, e.g., a partition principle (according to which one of any two natural kinds that are not necessarily disjoint is subordinate to the other), a summum genus principle (that every natural kind is subordinate to a natural kind that is not subordinate to any other natural kind), an infima species principle (that any natural kind of object belongs to a natural kind that is subordinate to every natural kind to which that object belongs), and various other principles (such as that the family of natural kinds to which an object belongs forms a chain of subordination such that each natural kind in the chain is a template structure that is causally more determinate and finer-grained than the natural kinds to which it subordinate). There are also methodological principles regarding the representation of natural kinds by sortal concepts. $^{43}$

It is clear, accordingly, without going into all the details here, that conceptualism can be analogically developed into a conceptual natural realism that is part of an Aristotelian essentialism, and that in this way it can account for various ontological categories or modes of being in the natural world of the space-time manifold that neither conceptualism alone nor conceptual idealism can account for.

\section{Conceptual Intensional Realism}

Frege's ontological insight that, by our logical faculties, we are able to grasp "and lay hold upon the extension of a concept by starting out from the concept" ([PW], p. 181), can be adapted to a conceptual intensional realism where, instead of extensions, we are conceptually able to grasp and lay hold upon the intensions of our concepts by starting out from those concepts. Historically, this grasp has come about through the evolution and institutionalization in language and culture of the rule-based process of nominalization. Conceptually, the process represents a kind of reflexive abstraction in which we attempt to represent what is not an object - e.g., an unsaturated cognitive capacity underlying our use of a predicate expression - as if it were an object. Thus, by means of such a reflexive abstraction, we can transform a predicate phrase, such as 'is wise', 'is triangular', 'is identical

\footnotetext{
${ }^{43}$ See Cocchiarella [1995] for a brief survey of both kinds of principles.
} 
with', into an abstract singular term, such as 'wisdom', 'triangularity', and 'identity', by which we purport to denote the intensional content of the concept the predicate stands for. Historically, it was Plato who first recognized the ontological significance of such a transformation, and who built his ontology around it.

Aristotle and Abelard, who are conceptual natural realists, rejected this kind of hypostitization. Abelard, for example, did think that a (conceptual/natural) universal such as being human was shared by, e.g., both Socrates and Plato, but he rejected the idea that such a universal could itself be a "thing", i.e. an object; and indeed it is not an object in the conceptual natural realism described above. The conceptual Platonist takes the opposite position, and assumes that the nominalization of a predicate expression, as an abstract singular term, denotes an abstract object, namely, the intensional content, or intension, of the concept that the predicate expression stands for in its role as a predicate. Such intensional objects are also typically called properties and relations, which of course should not be confused with the properties and relations of natural realism, and which should not be taken to mean that such objects have a predicable nature, which is true of the classes of Frege's ontology as well. Indeed, this kind of ontology is really a conceptual counterpart to Frege's logical realism, except that, unlike Frege, the conceptual Platonist does not take the principle of extensionality as a law of logic. Accordingly, our reconstruction of Frege's logic, i.e. the logical system $\mathrm{HST}_{\lambda}^{*}$ described in section 4 above (with or without modal operators and axioms included), can be taken as the logic of conceptual Platonism as well, which in some ways is preferable in that Frege's problem with predication does not apply to conceptual Platonism.

Conceptual Platonism is not the only way that the intensional contents of our concepts can be assumed to "exist" (or "be" the value of a bound individual variable), however. In what I have elsewhere called conceptual intensional realism, the Platonist idea that abstract objects "exist" outside of the causal order of space, time, and the evolution of consciousness is rejected. Abstract objects, on this account, have their being in the concepts whose intensions they are -just as Frege's classes are said to have their being in the concepts whose extensions they are (cf. [PW], p. 183). In conceptual Platonism, this is strictly an epistemological, and not an ontological, dependence. That is, it is only by starting out from concepts as cognitive capacities that we can have knowledge of abstract objects, i.e. knowledge of objects that, according to conceptual Platonism, "exist" in a realm that transcends space, time and causality, and therefore that "preexist" the evolution of consciousness and the very capacities by which they are grasped 
and understood. In conceptual intensional realism, on the other hand, the dependence is not merely epistemological but ontological as well. It is not only our grasp and knowledge of intensional objects that has come about through the evolution and development of the linguistic process of nominalization, i.e. the process whereby predicates and other expressions are transformed into abstract singular terms, but the very abstract being of those objects as well. It is in the evolution and institutionalization of this process, which began with the first rudimentary attempts to reflexively abstract the intensional content of our concepts - i.e. to reify, or "object-ify", the rule-based cognitive capacities that underlie our use of language - that the ultimate, explanatory ground of the mode of being of abstract objects is to be located.

Abstract objects are not only products of cultural evolution, on this account, but are also the means by which the further evolution of culture is possible. Thus, in addition to the abstract objects of mathematics, which are essential for the development of science and technology, there are also the intensional objects denoted by nominalized sentences, such as that-clauses, namely, propositions. Propositions are not the same as events and states of affairs, which are part of the causal order, but rather make up a "bracketed world" of intensional content within which we can freely speculate and make up theories about the natural world. Propositions also make up the content of our myths and stories, both true and fictional, and they serve in this way the literary and aesthetic purposes of culture. Fictional objects, in fact, are the intensional contents of the singular referential concepts that occur in a fiction - that is, they are the objects obtained by the conceptual counterpart of Frege's double-correlation thesis applied to the singular referential expressions that occur in fiction. ${ }^{44}$

The intensional logic, $\mathrm{HST}_{\lambda}^{*}$, supplemented with name variables and quantifiers restricted accordingly, can serve as the basis of a conceptual intensional realism such as is briefly indicated above - a framework that retains much of Frege's logic, but not his ontology. We can add to it the quantifiers $\forall^{n}$ and $\exists^{n}$, as well as $\forall^{k}$ and $\exists^{k}$, and the axioms and principles of natural realism and Aristotelian essentialism. The framework then is based primarily on a conceptual theory of predication together with a conceptual pattern of reflexive abstraction that reifies the content of our concepts. Through our conceptual activity we can formulate theories and hypothesize about whether or not there are natural properties and relations, or natural kinds, corresponding to certain of our predicable and sortal

\footnotetext{
${ }^{44}$ For a fuller account of the ontology of imaginary objects and fictional discourse, see Cocchiarella [1989b] and [1996].
} 
concepts. The general framework, which includes a conceptual intensional realism and a conceptual natural realism, can be simply called conceptual realism.

\section{Concluding Remarks}

Despite our extended discussion and defense of conceptual realism, the fact remains that this is a formal ontology that can be described and compared with other formal ontologies in the set-theoretic framework of comparative formal ontology. Set theory, as we have said, provides a convenient mathematical medium in which both the syntax and an extrinsic semantics of different formal ontologies can be formulated, which then can be compared and contrasted with one another in their logical and descriptive powers. This is the real insight behind the view of logic as calculus. But membership is at best a pale shadow of predication, which underlies thought, language and the different categories of reality. Set theory is not itself an adequate framework for general ontology, in other words, unless based on a theory of predication (as in Quine's nominalist-platonism). Only a formal theory of predication based on a theory of universals can be the basis of a general ontology. This is the real insight behind the view of logic as language. But there are alternative theories of universals, and therefore alternative formal theories of predication, each with its own logic and theory of logical form. A rational choice can be made only by formulating and comparing these alternatives in comparative formal ontology, a program that can best be carried out in set theory. Among the various alternatives that have been formulated and investigated over the years, the choice we have made here, for the reasons given, is what we have briefly described above as conceptual realism, which includes both a conceptual natural realism and a conceptual intensional realism. Others may make a different choice. As Rudolf Carnap once said: "Everyone is at liberty to build up his own logic, i.e. his own form of language, as he wishes." ${ }^{45}$ But then, at least in the construction of a formal ontology, we all have an obligation to defend our choice and to give reasons why we think one system is better than another. In this regard, we do not accept Carnap's additional injunction that "[i]n logic, there are no morals" (ibid.).

\footnotetext{
${ }^{45}$ Carnap [1937], §17.
} 


\section{References}

[[1965]] Addison, J.W., L. Henkin and A. Tarski, The Theory of Models (Amsterdam: North-Holland).

[1961] Bochenski, I.M., A History of Formal Logic (Notre Dame: University of Notre Dame Press).

[L\&O] Bochenski, I.M., "Logic and Ontology", Philosophy East and West, vol. 24 (1974): 275-292.

[1937] Carnap, R., The Logical Syntax of Language (London: Routledge \& Kegan Paul Ltd.), reprinted in 1951.

[1984] Chierchia, G., Topics in the Syntax and Semantics of Infinitives and Gerunds, Ph.D. dissertation (Amherst: University of Massachusetts).

[1985] Chierchia, G., "Formal Semantics and the Grammar of Predication", Linguistic Inquiry 16: 417-443.

[1987] Cocchiarella, N.B., Logical Studies in Early Analytic Philosophy (Columbus: Ohio State University Press).

[1988] Cocchiarella, N.B., "Predication Versus Membership in the Distinction between Logic as Language and Logic as Calculus", Synthesé 77: 37-72.

[1989a] Cocchiarella, N.B., "Philosophical Perspectives of Formal Theories of Predication", Handbook of Philosophical Logic, Vol. IV, edited by D. Gabbay and F. Guenthner (Dordrecht: D. Reidel Pub. Co.): 253-326.

[1989b] Cocchiarella, N.B., "Conceptualism, Realism, and Intensional Logic", Topoi 8: 15-34.

[1992] Cocchiarella, N.B., "Conceptual Realism Versus Quine on Classes and Higher-Order Logic", Synthesé 90: 379-436.

[1995] Cocchiarella, N.B., "Knowledge Representation in Conceptual Realism", International Journal of Human-Computer Studies 43: 697-721.

[1996] Cocchiarella, N.B., "Conceptual Realism as a Formal Ontology", Formal Ontology, edited by R. Poli and P. Simon (Dordrecht: Kluwer Academic Press). 
[1998] Cocchiarella, N.B., "Reference in Conceptual Realism", Synthese 114: 169-202.

[1954] Cohen, J., "On the Project of a Universal Character", Mind 63: 49-63.

[Gg] Frege, G., Die Grundgesetze der Arithmetik, vol.. 1 (Jena: Verlag Hermann Pohle, 1893); vols. 1 and 2 reprinted (Hildesheim: Georg Olms Verlagsbuchhandlung, 1962).

[1879] Frege, G., "Begriffsschrift, A Formula Language, Modeled upon that of Arithmetic, for Pure Thought", in From Frege to Gödel, edited by J. van Heijenoort (Cambridge: Harvard University Press, 1967).

[1972] Frege, G., Conceptual Notation and Related Articles, translated and edited by T.W. Bynum (Oxford: Oxford University Press).

[PW] Frege, G., Posthumous Writings, edited by Hermes, H., F. Kambartel, and F. Kaulbach (Chicago: Chicago University Press, 1979).

[R\&G] Geach, P., Reference and Generality, 1980, 3rd. edition (Ithaca: Cornell University Press).

[G\&B] Geach, P. and M. Black, eds., 1952, Translations From the Philosophical Writings of Gottlob Frege (Oxford: Blackwell).

[1956] Goodman, N., "A World of Individuals", in The Problem of Universals, a symposium with I.M. Bochenski, N. Goodman, and A. Church (Notre Dame: University of Notre Dame Press), pp. 15-31.

[1951] Goodman, N., The Structure of Appearance (Cambridge: Harvard University Press).

[1947] Goodman, N. and W.V.O. Quine, "Steps Toward a Constructive Nominalism", Journal of Symbolic Logic 12: 105-122.

[1988] Hintikka, J., "On the Development of the Model-Theoretic Tradition in Logical Theory", Synthesé 77: 1-36.

[1967] Kung, G., Ontology and the Logistic Analysis of Language (Dordrecht: D. Reidel). 
[1962] Lorenz, K., "Kant's Doctrine of the A Priori in the Light of Contemporary Biology", in General Systems, Yearbook of the Society for General Systems Research, Vol. VII, edited by L. von Bertalanffy and A. Rappaport, pp. 23-35.

[1974] Montague, R.M., Formal Philosophy, edited and an intro. by R. Thomason (New Haven: Yale University Press).

[1972] Piaget, J., The Principles of Genetic Epistemology (New York: Routledge and Kegan Paul).

[1963] Quine, W.V.O., Set Theory and its Logic (Cambridge: Harvard University Press).

[PoM] Russell, B., The Principles of Mathematics, second edition with a new intro. (New York: Norton \& Co., 1938).

[1967] van Heijenoort, J., "Logic as Language and Logic as Calculus" Synthesé 17: 324-330. 